\title{
Myocarditis: a defect in central immune tolerance?
}

\author{
Todd C. Metzger and Mark S. Anderson
}

Diabetes Center, UCSF, San Francisco, California, USA.

\begin{abstract}
Myocarditis, or inflammation of the heart, is a potentially devastating disease that can result from both viral infection and autoimmune attack of self antigens in the heart. In the current issue of the JCI, $\mathrm{Lv}$ and colleagues use a genetically susceptible mouse model to show that myocarditis is a $T$ cell-mediated autoimmune disease that occurs due to insufficient thymic negative selection of $\alpha$-myosin-reactive T cells.
\end{abstract}

\section{Myocarditis is a poorly understood disease}

Myocarditis is a disease characterized by the presence of inflammatory infiltrates in heart tissue, which can lead to dilated myocardiopathy and ultimately to congestive heart failure (1). Both viral and nonviral infections, such as those of coxsackie virus $B$ and Trypanosoma cruzi, respectively, are known to cause myocarditis (2). However, for a subset of patients with the disease, common causal infections are undetectable, but autoantibodies against cardiac antigens are present and symptoms improve following immunosuppressive treatments. Nevertheless, the physiological distinction between these two forms of myocarditis may be blurry, as some evidence supports a transition from a virally instigated heart inflammation to activation of the immune system against self antigens. Treatments for myocarditis remain insufficient, and further work toward identifying causes of myocarditis could help increase options. For example, identification of the relative importance of autoantigens in the more autoimmune forms of the disease could lead to antigen-specific tolerogenic therapies. Furthermore, the best diagnostic tool for myocarditis remains an invasive biopsy, so a better understanding of disease etiology could help guide less invasive diagnostic procedures.

\section{A spontaneous model of myocarditis}

The NOD mouse contains many genetic susceptibility loci that cause it to spontaneously develop autoimmune diabetes in a $\mathrm{T}$ cell-dependent manner (3). In 2003, it was found that replacement of a specific allele of the MHC class II complex (I-Ag7) with a human DQ8 allele (DQ8 ${ }^{+}$NOD mice)

Conflict of interest: The authors have declared that no conflict of interest exists.

Citation for this article: J Clin Invest. doi:10.1172/ JCI57211. led to protection from NOD autoimmune diabetes, but also resulted in a predisposition to myocarditis and dilated cardiomyopathy (4). Paradoxically, the human DQ8 allele had previously been associated with type 1 diabetes. Nevertheless, further investigation showed that dilated cardiomyopathy in these mice was a result of $\mathrm{CD} 4^{+} \mathrm{T}$ cell activity and that autoantibodies alone were insufficient to account for the observed disease $(5,6)$. Thus, while the DQ8 ${ }^{+} \mathrm{NOD}$ mouse strain is a valuable model for studying $\mathrm{T}$ cell-mediated autoimmunity arising from multiple dysfunctional regulatory pathways, the mechanism directly responsible for disease development in these mice remained unknown.

In the current issue of JCI, Lv et al. demonstrated that a defect in thymic negative selection, the deletion of autoreactive T cells during their development, is responsible for the spontaneous myocarditis observed in DQ8 ${ }^{+}$NOD mice. The authors showed that the presence of target antigen for $\alpha$-myosin-specific $T$ cells in the thymus prevented their functional maturation and attack of cardiac tissue (7). First, the authors showed that it is the cardiac-specific $\alpha$-myosin isoform, and not the skeletal muscle $\beta$-myosin isoform, that appears as the first autoantibody target in DQ8 ${ }^{+} \mathrm{NOD}$ mice, consistent with the heart-directed autoimmunity observed in these mice. They also demonstrated that $T$ cells specific for $\alpha$-myosin could be isolated from heart infiltrates, and that such $T$ cells displayed a potentially pathogenic Th1 cytokine profile. Next, the authors examined the $\mathrm{B}$ and $\mathrm{T}$ cell dependence of DQ8 ${ }^{+} \mathrm{NOD}$ myocarditis using mice selectively deficient in either cell type, and found that while $T$ cells are critical for disease to occur, myocarditis could occur in the absence of B cells, albeit at a slower rate. This mirrors the relative $\mathrm{T}$ and $\mathrm{B}$ cell dependence of autoimmune diabetes development in NOD mice and is consistent with autoimmune disease resulting primarily from defective tolerance among T cells (3). Interestingly, this finding also shares similarities to observations of the Aire-knockout mouse, in which defective negative selection of autoreactive $\mathrm{T}$ cells in the thymus leads to multiorgan autoimmune disease mediated exclusively by $\mathrm{T}$ cells (8).

\section{Thymic selection prevents myocarditis in DQ8+NOD mice}

Having provided evidence for the importance of $\alpha$-myosin as a $T$ cell antigen in DQ8 ${ }^{+}$NOD myocarditis, Lv et al. went on to examine whether defective thymic selection and escape of $\alpha$-myosin-reactive T cell clones from the thymus might explain why DQ8 ${ }^{+}$NOD mice develop myocarditis (7). After finding that $\alpha$-myosin mRNA seemed to be nearly undetectable in medullary thymic epithelial cells (mTECs) by examining published array data, the authors confirmed by quantitative PCR that $\alpha$-myosin is unique among examined cardiac antigens in its absence from the thymic stroma. Suspecting that this lack of thymic antigen allowed for the development of $\alpha$-myosin-reactive $\mathrm{T}$ cells and subsequent heart infiltration in DQ8 ${ }^{+}$NOD mice, the authors transgenically introduced $\alpha$-myosin into the thymus of DQ8 ${ }^{+}$NOD mice and observed complete protection from the development of myocarditis (Figure 1). Furthermore, they found that peripheral $\mathrm{T}$ cells from mice with thymic $\alpha$-myosin remained unresponsive to the antigen ex vivo, which, taken together with the prevention from myocarditis, showed that the thymic antigen effectively tolerizes DQ8 ${ }^{+}$NOD mice against $\alpha$-myosin-directed autoimmunity. Last, the authors suggested that central tolerance to $\alpha$-myosin may be ineffective in humans. They demonstrated that expression of $\alpha$-myosin is near the minimum level of detection in stromal cells of the human thymus, meaning that it may be of little functional consequence. Ex vivo assays with peripheral blood mononuclear leukocytes demonstrated that while control patients exhibited some responsiveness to $\alpha$-myosin even in the absence of disease, the response was markedly increased in patients with inflammatory heart disease. As relatively few patient samples were analyzed, 

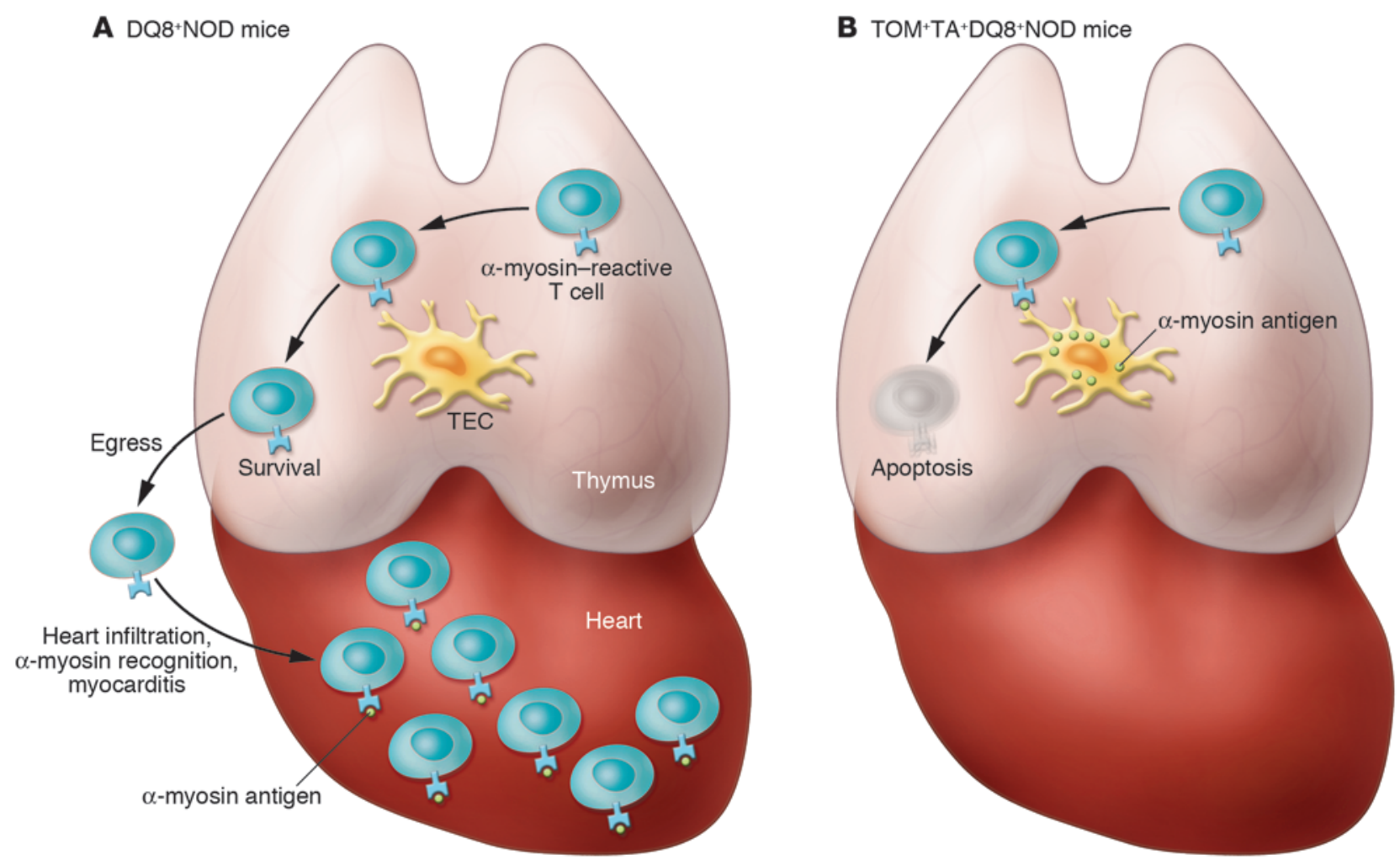

\section{Figure 1}

Permissive negative selection leads to myocarditis in DQ8+NOD mice. In DQ8+NOD mice (A), $\alpha$-myosin-reactive T cells escape the thymus, presumably due to the absence of negatively selecting $\alpha$-myosin antigen within the thymus. These T cells are then able to recognize their target antigen within the heart and heart-draining lymph nodes, causing inflammation and myocarditis. When $\alpha$-myosin is transgenically introduced into thymic epithelial cells (TEC) of this same mouse strain (B; TOM+TA+DQ8+NOD mice), negative selection likely induces deletion of developing $\alpha$-myosin-reactive T cells, preventing their maturation, egress to the periphery, and infiltration of the heart.

further study is warranted to determine whether such assays will be broadly applicable to patients with myocarditis.

\section{The mechanism remains unclear}

The findings of this article clearly demonstrate the importance of central tolerance in preventing autoreactive $T$ cell development and subsequent autoimmunity, and provide another example of the value of studying models of spontaneous autoimmunity to identify autoantigens with clinical relevance (9). However, a lack of central tolerance to $\alpha$ myosin in DQ8 ${ }^{+} \mathrm{NOD}$ mice does not entirely explain the unique susceptibility of this mouse strain to myocarditis. WT NOD mice also lack $\alpha$-myosin expression in the thymus, but, in these mice, the resulting lack of negative selection and release of $\alpha$-myosin-reactive $T$ cells to the periphery do not result in myocarditis. Therefore, the underlying cause of myocarditis induction in DQ8 $8^{+} \mathrm{NOD}$ is likely to be peculiarities with the binding of $\alpha$-myosin-derived peptides to the introduced DQ8 MHC allele, which might affect process- es that are sufficient to mediate tolerance in WT NOD mice. Also, despite the absence of substantial $\alpha$-myosin transcripts among thymic stroma, central tolerance to this antigen may still be physiologically relevant in DQ8 ${ }^{+}$NOD mice, as recirculating dendritic cells, which pick up antigen in the periphery and then traffic to the thymus, have been shown to be an effective source of antigen for negative selection (10). When T cells encounter their antigen in peripheral tissues under non-inflammatory conditions, they are likely to be tolerized, so encounter of $\alpha$-myosin-specific $T$ cells with their antigen in heart-draining lymph nodes is a probable mechanism of tolerance in WT NOD mice. However, these tolerance processes are sensitive to the strength of signal delivered by antigen-MHC complexes, so differences in antigen binding and presentation by the DQ8 allele might perturb this mechanism of tolerance. Similarly, the introduced DQ8 allele is expressed as a transgene, as opposed to a knock-in, and, as such, may be expressed at levels that differ from those of endogenously expressed MHC class II complexes in WT NOD mice. Finally, the mechanism by which central tolerance is established in mice expressing transgenic thymic antigen is unclear, as negative selection of autoreactive $T$ cells and induction of immunosuppressive regulatory $\mathrm{T}$ cells might both contribute to disease protection in this experimental system.

\section{Future directions}

The findings of the article by Lv et al. (7) demonstrate that, at least in $\mathrm{DQ} 8{ }^{+} \mathrm{NOD}$ mice, $\alpha$-myosin is the earliest detectable target of autoantibodies in myocarditis, suggesting that it is likely an important initiating autoantigen for the autoimmune form of the disease. Further work is warranted to determine whether this autoantigen may have good early predictive value of future autoimmune cardiomyopathy in clinical settings. Also, given the ability of $\alpha$-myosindirected central tolerance to completely protect mice from myocarditis, future therapeutic approaches in the clinical arena may seek to induce $\mathrm{T}$ cell tolerance to this antigen. 


\section{Acknowledgments}

This work was supported by the Juvenile Diabetes Research Foundation (JDRF), NIH, Leona M. and Harry B. Helmsley Charitable Trust, and Burroughs Wellcome Fund. We would also like to thank Kellsey Johannes and Imran Khan for helpful comments on a draft of this commentary.

Address correspondence to: Mark S. Anderson, UCSF Diabetes Center, 513 Parnassus Ave. HSW 1114, Box 0540, San Francisco, California 94143, USA. Phone: 415.502.8052; Fax: 415.564.5813; E-mail: manderson@diabetes.ucsf.edu.
1. Cooper LT. Myocarditis. N Engl J Med. 2009; 360(15):1526-1538

2. Rose NR. Myocarditis: infection vs. autoimmunity. J Clin Immunol. 2009;29(6):730-737.

3. Anderson MS, Bluestone JA. The NOD mouse: a model of immune dysregulation. Annu Rev Immunol. 2005;23:447-485.

4. Elliott JF, et al. Autoimmune cardiomyopathy and heart block develop spontaneously in HLA-DQ8 transgenic IA $\beta$ knockout NOD mice. Proc Nat Acad SciUS A. 2003;100(23):13447-13452.

5. Taylor JA, Havari E, McInerney MF, Bronson R, Wucherpfennig KW, Lipes MA. A spontaneous model for autoimmune myocarditis using the human MHC molecule HLA-DQ8. I Immunol. 2004;172(4):2651-2658.

6. Hayward SL, Bautista-Lopez N, Suzuki K, Atrazhev A, Dickie P, Elliott JF. CD4 T cells play major effector role and CD8 T cells initiating role in spon- taneous autoimmune myocarditis of HLA-DQ8 transgenic IAb knockout nonobese diabetic mice. J Immunol. 2006;176(12):7715-7725.

7. $\mathrm{Lv} \mathrm{H}$, et al. Impaired thymic tolerance to $\alpha$-myosin directs autoimmunity to the heart in mice and humans. J Clin Invest. 2011;121(4):1561-1573.

8. DeVoss JJ, et al. Effector mechanisms of the autoimmune syndrome in the murine model of autoimmune polyglandular syndrome type 1.J Immunol. 2008;181(6):4072-4079

9. Shum AK, et al. Identification of an autoantigen demonstrates a link between interstitial lung disease and a defect in central tolerance. Sci Transl Med. 2009;1(9):9ra20.

10. Bonasio R, Scimone ML, Schaerli P, Grabie N, Lichtman AH, von Andrian UH. Clonal deletion of thymocytes by circulating dendritic cells homing to the thymus. Nat Immunol. 2006; 7(10):1092-1100

\title{
Niche competition and cancer metastasis to bone
}

\author{
Laura G. Schuettpelz ${ }^{1}$ and Daniel C. Link ${ }^{2}$ \\ 1Division of Hematology/Oncology, Department of Pediatrics, and 2Division of Oncology, Department of Medicine, \\ Washington University School of Medicine, St. Louis, Missouri, USA.
}

\begin{abstract}
The molecular basis for the preferential metastases of certain cancers to bone is not well understood. In this issue of the JCI, Shiozawa et al. provide compelling evidence that prostate cancer cells preferentially home to the osteoblastic niche in the bone marrow, where they compete with normal HSCs for niche support. Because signals from the niche may regulate tumor quiescence and sensitivity to chemotherapy, these observations have important implications for the treatment of metastatic prostate cancer in bone.
\end{abstract}

Bone is a common site of metastases for certain tumors, including breast cancer and prostate cancer ( $\mathrm{PCa}$ ). Approximately $70 \%$ of patients with $\mathrm{PCa}$ have bone metastases at the time of death (1). The molecular basis for this preferential growth in the bone marrow and the biological effects of the rich microenvironment in the bone marrow on cancer cell growth and survival are not well understood. In this issue, Shiozawa et al. provide compelling evidence that PCa cells preferentially home to the osteoblastic niche in the bone marrow, where they compete with normal HSCs for niche support (2). Because signals from the niche may regulate the quiescence and survival of PCa cells (and possibly sensitivity to chemotherapy), these observations have important implications for the treatment of metastatic bone cancer.

The bone marrow microenvironment plays a critical role in the maintenance

Conflict of interest: The authors have declared that no conflict of interest exists.

Citation for this article: J Clin Invest. doi:10.1172/ JCI57229. of HSC quiescence and self-renewal. HSCs preferentially localize in the bone marrow, either to a perivascular location or near the endosteum $(3,4)$. Although the stem cell niche in the bone marrow is likely to be complex, with contributions from endothelial cells, advential reticular cells, nestinpositive stromal cells (5), and CXCL12abundant reticular (CAR) cells $(6,7)$, current evidence suggests that osteoblast lineage cells are a key component of the endosteal niche and are required to maintain normal HSC function. Expansion of osteoblast lineage cells by genetic or pharmacologic means results in concurrent expansion of HSCs (8). Conversely, ablation of osteoblasts using a suicide gene results in a loss of HSCs (9).

\section{CXCL12/CXCR4 axis}

Although the signals generated by the stem cell niche that regulate HSCs are not fully understood, a key player is CXCL12 (also known as stromal-derived factor-1), a chemokine constitutively expressed at high levels in the bone marrow by osteo- blasts, endothelial cells, and other bone marrow stromal cells. CXCL12, primarily through interaction with its major receptor, CXCR4, regulates HSC quiescence and homing to the bone marrow $(10,11)$. Disruption of CXCL12/CXCR4 signaling is a key step in cytokine-induced HSC mobilization from bone marrow to blood (12). The importance of the CXCL12/ CXCR4 axis is shown by the success of the CXCR4 inhibitor plerixafor (AMD3100; Genzyme) to rapidly mobilize HSCs in humans (13). Other agents produced by stromal cells in the endosteal niche that have been implicated in the regulation of HSCs include angiopoietin-1, thrombopoietin, and mediators of Notch and Wnt signaling (14-16).

Certain tumors, including PCa cells, appear to have coopted the CXCL12/ CXCR4 signaling pathway to preferentially home to the bone marrow. Whereas CXCR4 expression is low or absent in many normal tissues, it is expressed at high levels in more than 23 different cancers, including breast cancer, ovarian cancer, and PCa (17).

Importantly, inhibition of CXCR4 signaling has been shown to reduce metastatic disease of multiple tumor types in mouse xenograft models. The growth and metastasis of PCa cells injected into nude mice, for example, was inhibited by a neutralizing antibody to CXCR4 (15). Similarly, treatment of mice with small-molecule 